\title{
An Improved Information Hiding Method Based on Sparse Representation
}

\author{
Minghai Yao, ${ }^{1,2,3}$ Miao Qi, ${ }^{1}$ Yugen Yi, ${ }^{1}$ Yanjiao Shi, ${ }^{1}$ and Jun Kong ${ }^{1,2}$ \\ ${ }^{1}$ School of Computer Science and Information Technology, Northeast Normal University, Changchun 130017, China \\ ${ }^{2}$ School of Mathematics and Statistics, Northeast Normal University, Changchun 130017, China \\ ${ }^{3}$ College of Information Science and Technology, Bohai University, Jinzhou 121013, China
}

Correspondence should be addressed to Miao Qi; qim801@nenu.edu.cn and Jun Kong; kongj435@nenu.edu.cn

Received 5 November 2014; Revised 9 December 2014; Accepted 9 December 2014

Academic Editor: Hui Zhang

Copyright (c) 2015 Minghai Yao et al. This is an open access article distributed under the Creative Commons Attribution License, which permits unrestricted use, distribution, and reproduction in any medium, provided the original work is properly cited.

\begin{abstract}
A novel biometric authentication information hiding method based on the sparse representation is proposed for enhancing the security of biometric information transmitted in the network. In order to make good use of abundant information of the cover image, the sparse representation method is adopted to exploit the correlation between the cover and biometric images. Thus, the biometric image is divided into two parts. The first part is the reconstructed image, and the other part is the residual image. The biometric authentication image cannot be restored by any one part. The residual image and sparse representation coefficients are embedded into the cover image. Then, for the sake of causing much less attention of attackers, the visual attention mechanism is employed to select embedding location and embedding sequence of secret information. Finally, the reversible watermarking algorithm based on histogram is utilized for embedding the secret information. For verifying the validity of the algorithm, the PolyU multispectral palmprint and the CASIA iris databases are used as biometric information. The experimental results show that the proposed method exhibits good security, invisibility, and high capacity.
\end{abstract}

\section{Introduction}

In recent years, with the rapid development of the information technology, Internet has become an indispensable part of people's lives. In the meantime, Internet fraud and network attack have become a major problem to Internet users. Network service system puts forward higher requirements for the accuracy, security, and reliability of identity recognition. The traditional identity recognition methods, such as smart card, ID, and password, have been unable to meet the need of people. Biometric characteristics of the human body, such as palmprint, iris, and face, have the properties of uniqueness and invariability, which have become an important foundation for identity recognition.

The biometrics is a new identification technique. An individual is identified by their distinct physiological or behavioral characteristics. The identification method based on biometric technology is better than traditional methods. However, biometric data itself has no confidentiality and security. Therefore, the security problem of biometric data has already become an urgent and important problem. Information hiding is an effective solution to protect security and integrity of biometric data. Many researchers have proposed various methods for protecting biometric data.

Bedi et al. presented the multimodal biometric authentication method using PSO based watermarking [1]. The key idea is that the multimodal biometric image was used as the watermark image. PSO was used to select best DCT coefficient in the face image. Vatsa et al. presented a three-level RDWT biometric watermarking algorithm for embedding the voice biometric MFC coefficient into a color face image [2]. The watermarking algorithm used adaptive user-specific parameters for improving performance. A novel method of empirical mode decomposition and gene expression programming together was used to embed biometric information in the literature [3]. The singular value decomposition and lifting based discrete wavelet transform were employed in the watermarking algorithm. $\mathrm{Li}$ et al. proposed Tamper 


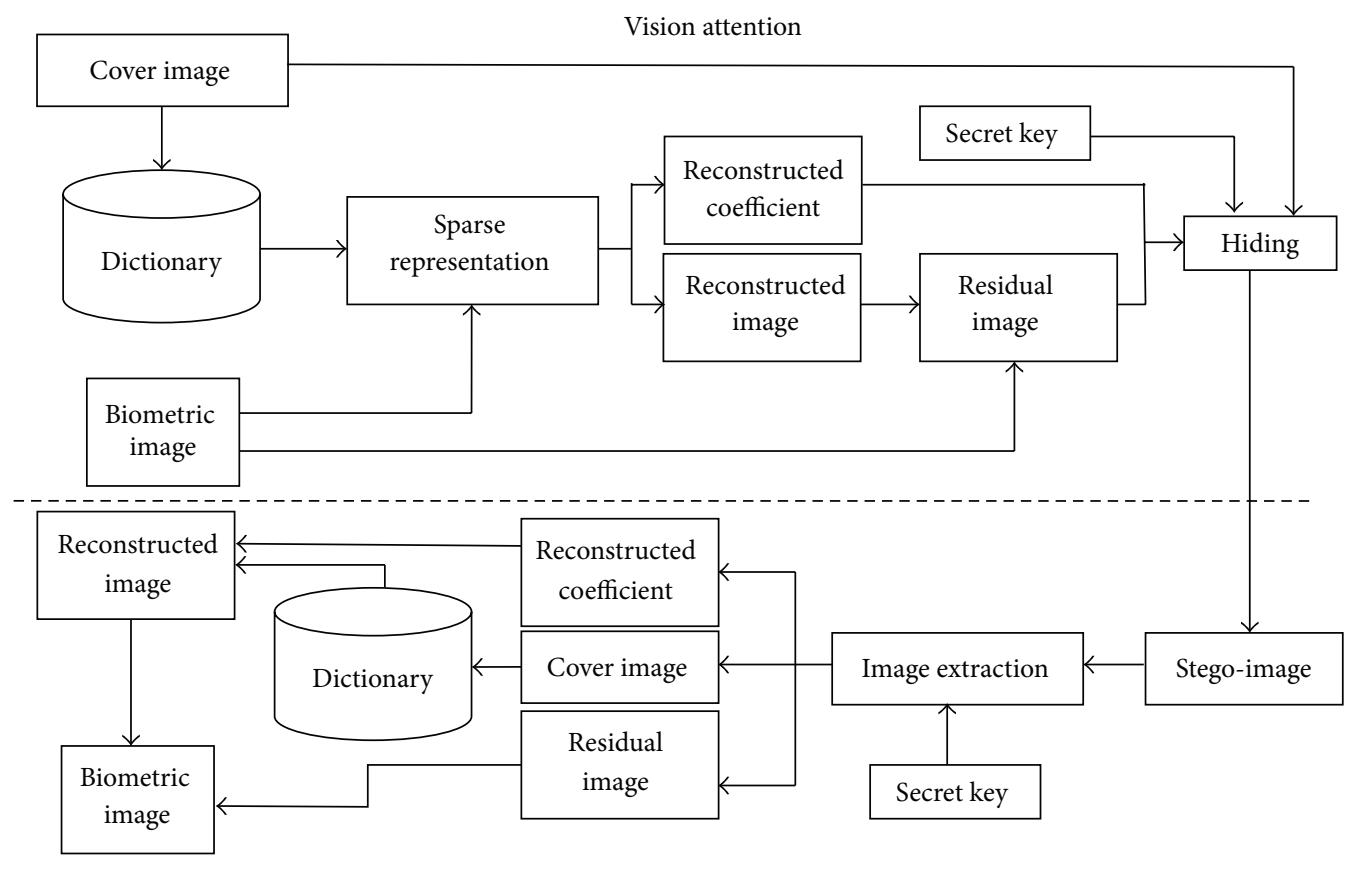

FIGURE 1: The flowchart of biometric information hiding method.

detection and self-recovery of biometric images using salient region-based authentication watermarking scheme [4]. Che et al. took into account the content relevance of the cover image and the watermarked image; they proposed contentbased image hiding method [5]. The biometric information hiding methods based on correlation analysis were proposed in the literature [6]. For the video data, the dual video watermark authentication method has been proposed by Shi et al. [7].

Through analyzing existing biometric information hiding methods, we found almost all of hiding methods adopt digital watermarking and hide one or more biometric images or their features into another biometric image directly based on transform domain for verification. These methods are robust against some types of attacks, but the hiding capacity is low. At the same time, the existing methods rarely consider the content correlation between the biometric image and the cover image. The cover image is only used as a hidden carrier and their rich content cannot be fully utilized. Therefore, a novel biometric authentication image hiding method based on the sparse representation is proposed in this paper, which considers the content correlation between the biometric image and the cover image adequately.

\section{The Proposed Method}

The sparse representation and visual attention model are used in the biometric hiding method, which uses the sparse representation theory to analyze the content correlation between the cover image and the biometric image. First, the dictionary is built by the cover images for calculating the sparse representation coefficients of a biometric image. The biometric image is reconstructed by the dictionary and sparse representation coefficients. The difference between the original biometric image and the reconstructed biometric image is used as one part of the secret information. The sparse representation coefficients are another part of the secret information. The two parts of secret information are embedded into the cover image. In order to facilitate the embedding of secret information, the secret information is converted into a binary sequence. Through statistical analysis of a large number of experimental data, we found that the absolute value of each pixel value in the residual image is less than 31. Therefore, each pixel value of residual image can be represented by six binary bits, where the first one represents the sign bit and the other five are the pixel values. The amount of embedded information is greatly reduced compared with the original image. Besides, the embedding region of secret information is selected by the saliency image of cover image. At last, the reversible watermarking algorithm based on histogram is used to embed secret information, where the embedded order is determined by the saliency image of the cover image.

When we extract the secret information, the binary sequence in stego-image is firstly extracted by the secret key. Then, the binary sequence is converted to residual image and sparse representation coefficients. The dictionary is built by recovered stego-image. Finally, the biometric image is restored by the residual image and the reconstructed biometric image.

The flowchart of hiding method is described in Figure 1. The method is divided into three stages of sparse representation, vision attention, and image hiding.

2.1. Sparse Representation. With the rapid development of computer technology, abundant signal processing methods 
have been proposed. As a branch of signal processing, sparse representation has been wildly applied in image denoising, image restoration, feature extraction, image compression, pattern recognition, machine learning, compressed sensing, and other fields. Nevertheless, the sparse representation theory is rarely used in the information hiding field. Jost et al. applied the sparse representation theory to the information security field; the secret information was embedded into the decomposition path of the sparse decomposition image. The receiver extracts the secret information by decomposition path of the cover image [8]. Cencelli and his colleagues embedded information by modifying the sparse representation coefficients of image [9-11]. These methods only use a sparse representation coefficient and decomposition path to achieve information hiding. They do not analyze the correlation between the secret information and the cover image by sparse representation method. Therefore, this paper uses sparse representation method to analyze the correlation between secret information and the cover image for reducing the number of secret information.

The element of decomposition signal is called the atom in the sparse representation theory. Suppose $y \in R^{n}$ is an image block representation vector, $D \in R^{n \times L}$ represents redundant dictionary, $L$ represents the number of atoms, and sparse representation process can be described as

$$
\widehat{\alpha}=\underset{\alpha}{\arg \min }\|y-D \alpha\|_{2}^{2} \quad \text { s.t. }\|\alpha\|_{0} \leq S,
$$

where $\|*\|_{2}$ represents the $l_{2}$-norm, $\|*\|_{0}$ represents the $l_{0}$ norm, $S$ represents sparse degree, $D$ is the redundant dictionary, $D=\left[d_{1}, d_{2}, \ldots, d_{L}\right], \alpha$ represents sparse representation coefficient, and $\|\alpha\|_{0}$ represents the number of non-zero coefficients in $\alpha$. Figure 2 is schematic diagram of the sparse representation theory.

Two of the most critical problems need to be solved using the sparse representation for information hiding. One is how to build an effective dictionary $D$, and the other is how to obtain sparse representation coefficient. Taking into account the characteristics of the cover image and biometric image, we use the integer value dictionary. In order to reduce the difficulty and the complexity of building dictionary, data sampling method is used to build the redundant dictionary by the cover image. Palmprint images and iris images are divided into blocks for improving the computational efficiency of the algorithm in this paper. Palmprint image is divided into 16 blocks with the size of $32 \times 32$; iris image is divided into 8 blocks with the size of $32 \times 32$. The dictionary is built by data sampling methods with size of $1024 \times 6561$. Orthogonal matching pursuit algorithm (OMP) is used to calculate the sparse representation coefficient [12] which is an improved algorithm based on matching pursuit algorithm [13]. The original image, reconstructed image and residual image are shown in Figures 3 and 4.

2.2. Vision Attention. Visual attention mechanism is an emerging research field, which contains neurobiology psychology, computer vision, pattern recognition, artificial intelligence, and many other disciplines. It is one kind of mechanism of human visual systems in selecting regions of interest

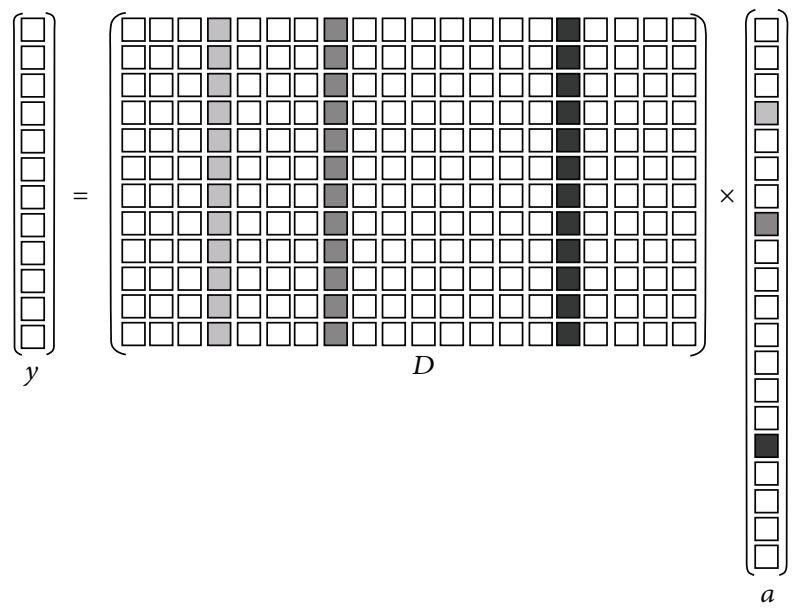

Figure 2: The schematic diagram of the sparse representation theory.

from complex scenes. Recently, it has become the focus of research in computer vision, due to its applications for object detection [14-16] and digital image processing field [17-20]. Visual attention model is divided into two categories by visual information processing method in computer vision system. The first one is a bottom-up visual attention model method, which is directed by the data. But it is not dependent on the specific task. The second one is a top-down visual attention model method, which is affected by subjective consciousness. It depends on the specific task. In this paper, we use the method of literature [21]. The saliency of object is detected by the unified approach, which integrates bottom-up for lowerlevel features and top-down for higher-level priors.

After multiscale feature extraction, we decompose an image into small regions by image segmentation. Then the mean of the feature vectors in a segment is treated as the feature. The matrix representation of the image is formed by stacking them. By this means, even when the object size is large, the number of segments in a salient object is still small, due to the fact that salient objects usually have spatial and appearance-wise coherence. At the same time, in order to ensure that the matrix representing the background has a low rank, we train a linear feature transformation using labeled data. This method can achieve good performance on saliency detection, even without higher-level knowledge. Figures 5 and 6 show the illustration of the method.

In order to ensure the consistency of the saliency image from the cover image and the stego-image, the saliency image is computed by the reference subsampling image. The reference subsampling image is computed by (3) and (4).

2.3. Information Hiding. One target of information hiding is to hide secret information into another nonsecret cover image for avoiding the attacker's attention. The information hiding technique requires that the secret information cannot be found in digital media. At the same time, if the attacker does not get the secret key, anyone cannot extract the secret information from the digital media. 


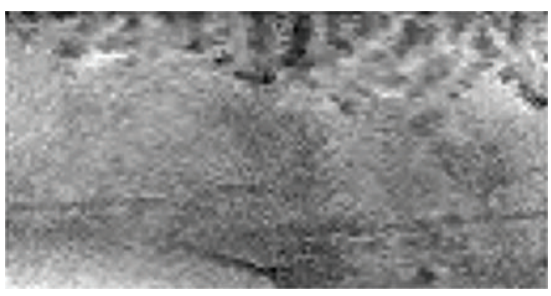

Original image

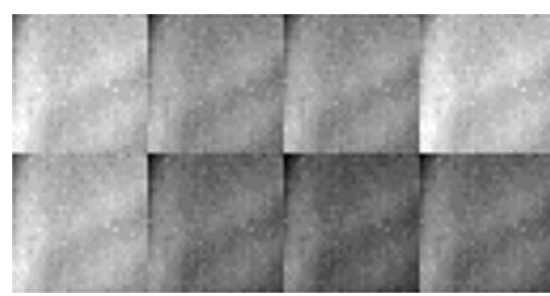

Sparse representation results

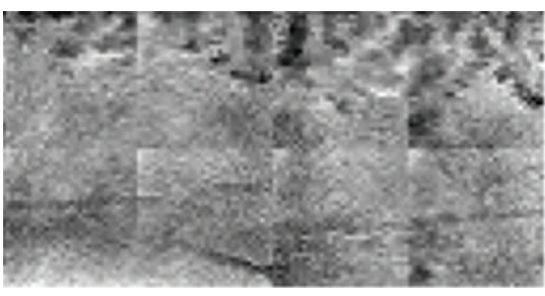

Residual image

FIGURE 3: Iris image reconstruction result.

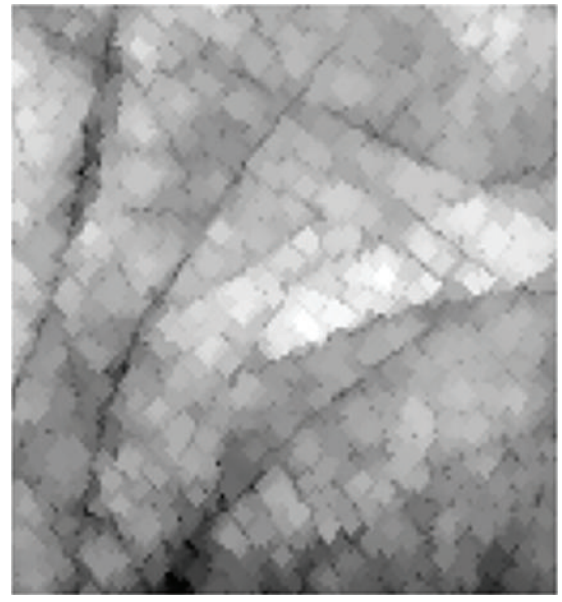

Original image

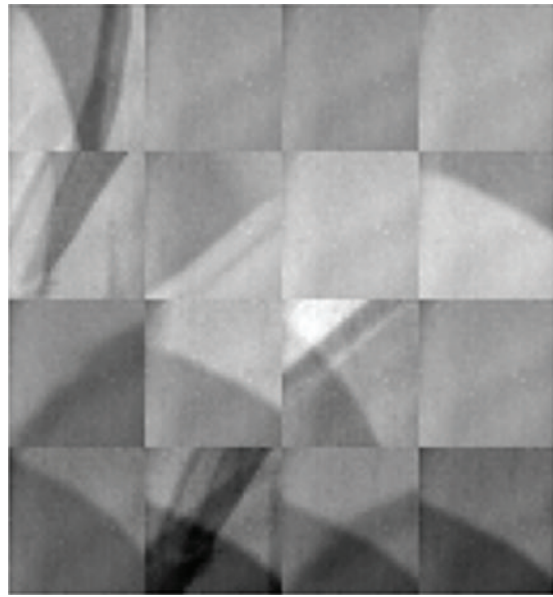

Sparse representation results

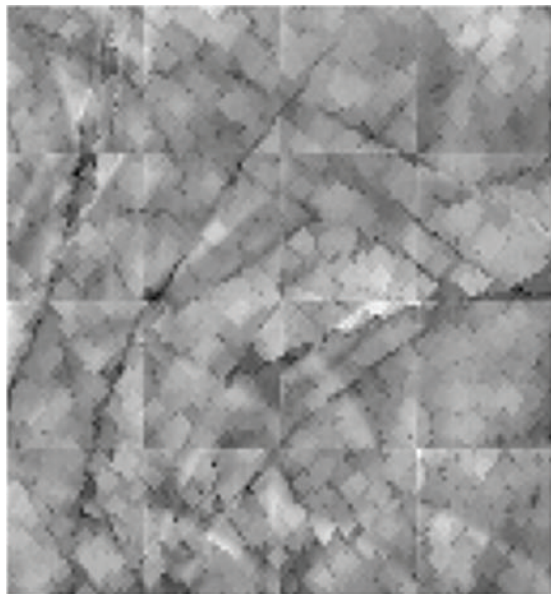

Residual image

FIgURE 4: Palmprint image reconstruction result.

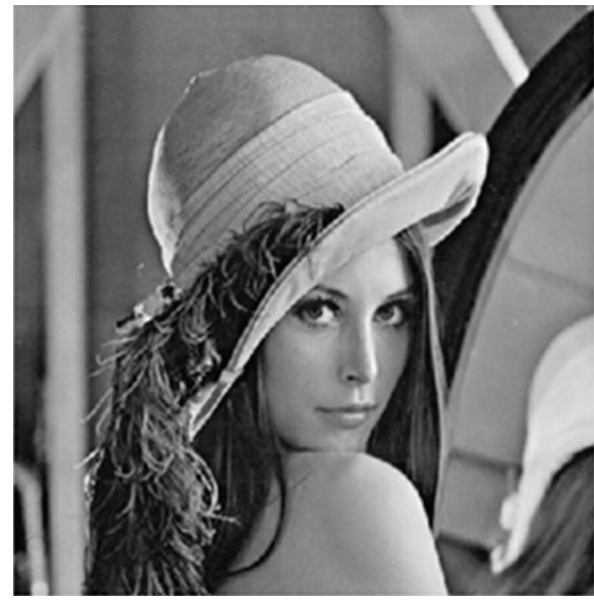

Cover image

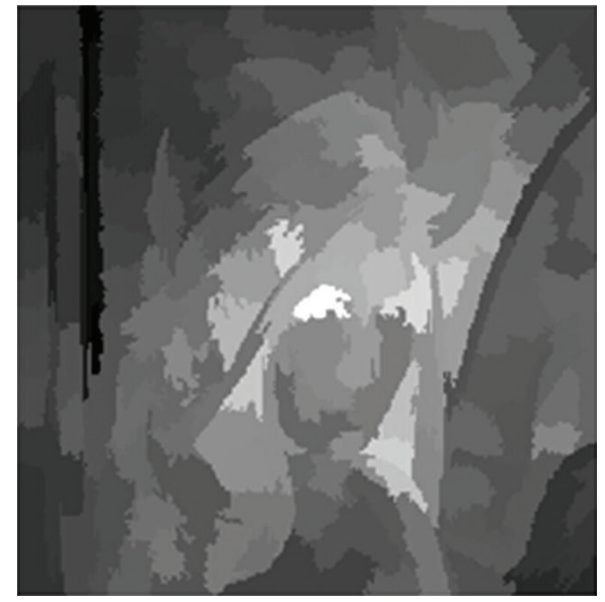

Image saliency

FIGURE 5: Lena image and its saliency image.

The histogram is the most basic statistical characteristics of the image. A digital image has $L$ gray-level in the range $[0, G]$, and the discrete function of the histogram is defined as

$$
h(k)=n_{k},
$$

where $k$ represents the gray value of the image, $n_{k}$ represents the number of the pixel that the gray value is $k$, and the value of $G$ is 255 in the gray image.

Histogram analysis is an important tool for digital image processing. Thus, the reversible watermarking algorithm based on histogram is used in the process of embedding secret 

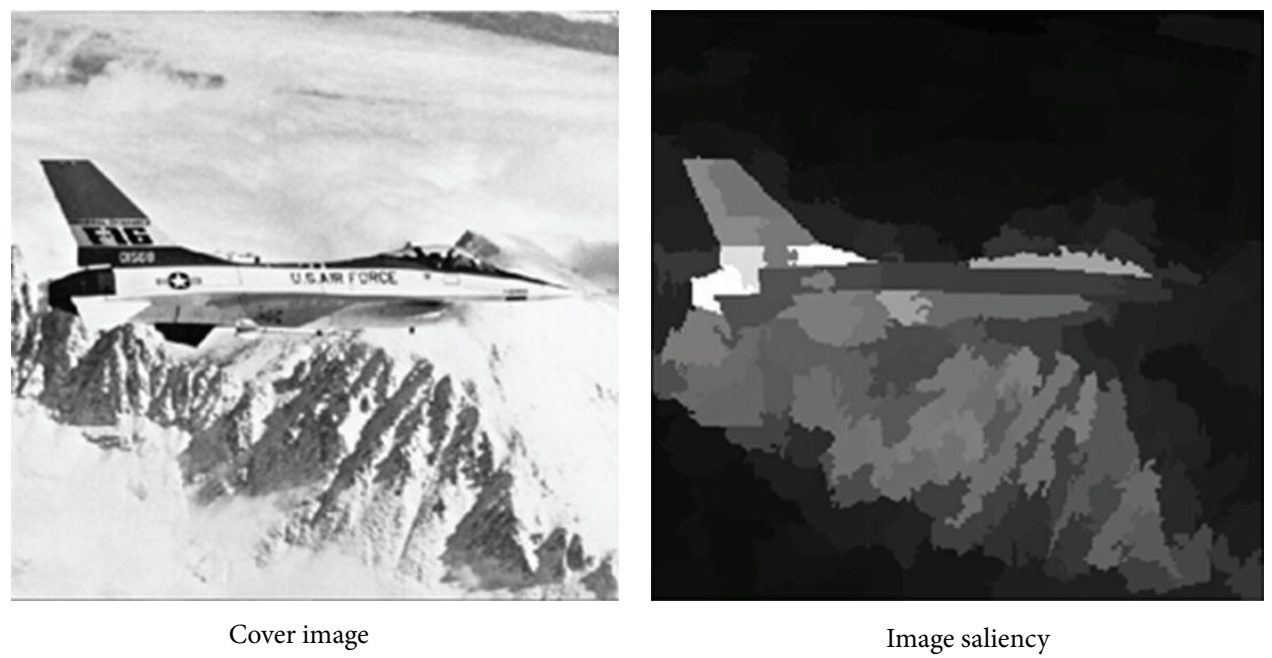

FIGURE 6: Airplane image and its saliency image.

information. The research focus of the traditional hiding algorithm based on histogram is how to determine the peakvalue point and zero-value point, which results in that the watermark embedding capacity is too small and random. The application scope of the algorithm is limited because of these problems. A large number of statistical results show that the embedding capacity can be increased by segmenting the image into blocks. Since the pixel value is relatively concentrated in the image block, more embedded space can be got from the image [22].

The watermark embedding method of literature [23] is used in this paper, meanwhile the division block method of cover image and embedding sequence of watermark are improved. The cover image is divided into small pieces by saliency image, each of which is a small cover image. All image blocks are sorted by significance. The secret information is segmented according to the embedding capacity of each small cover image and embedded in the corresponding cover image block until all the secret information is embedded.

The embedding method of secret information can be described as follows.

Step 1. According to the significance, the block of cover image $I$ is selected, and the values of sampling coefficients $u$ and $v$ are 2 , respectively. All the subsampled images $I_{1}, I_{2}, I_{3}, I_{4}$ are generated by (3)

$$
I_{m}(i, j)=I\left(i \cdot v+\text { floor }\left(\frac{m-1}{u}\right), j \cdot u+(m-1) \bmod u\right) .
$$

Step 2. $I_{\text {Ref }}$ represents the reference subsampled image. $I_{\text {Des }}$ represents the target subsampled image. $I_{\text {Ref-Des }}$ represents the difference of $I_{\text {Ref }}$ and $I_{\text {Des }}$. All $I_{\text {Ref-Des }}$ are calculated by (5):

$$
\begin{gathered}
I_{\mathrm{Ref}}=\left(\operatorname{Round}\left(\frac{u}{2}-1\right)\right) \times v+\operatorname{Round}\left(\frac{v}{2}\right), \\
I_{\text {Ref-Des }}(i, j)=I_{\text {Ref }}(i, j)-I_{\text {Des }}(i, j)
\end{gathered}
$$

where $0 \leq i \leq M /(v-1), 0 \leq j \leq N /(u-1)$.
Step 3. The embedding position of secret information is determined by moving the histogram based on the embedded level $L$. In order to adaptively embed secret information, the histogram is moved around according to embedding level $L$. When the histogram is modified, the secret information is embedded in the range of $[-L, L]$. The moving method of the histogram is shown in the following:

$$
H_{S}= \begin{cases}H+L+1 & H \geq L+1 \\ H-L-1 & H \leq-L-1\end{cases}
$$

where $H$ represents the gray values and $L$ represents embedding level.

The pixel value of the reference subsampling image cannot be changed in order to ensure reversibility of the method. Therefore, we can only modify the pixel value of the target subsampled image. The modification method of the pixel value is shown in the following:

$$
I_{\text {Des }}^{\prime}(i, j)= \begin{cases}I_{\text {Des }}(i, j)-(L+1) & H \geq L+1 \\ I_{\text {Des }}(i, j)+(L+1) & H \leq-L-1 .\end{cases}
$$

Step 4. The secret information is embedded by moving the histogram. We scan the pixel value of $I_{\text {Ref-Des }}$. When the size of the pixel value is $L$ or $-L$, the secret information is embedded. The scanning process is repeated until all the pixel values are not equal to $L$ or $-L$. In this case, the embedded level $L$ is -1 , and repeat the above process until $L<0$. The embedding method is shown in (8). The moving process of the histogram is shown in Figure 7:

$$
L>0
$$

$$
I_{\text {Des }}^{\prime \prime}(i, j)= \begin{cases}I_{\text {Des }}^{\prime}(i, j)-(L+1) & P^{\prime}=L, w(n)=1 \\ I_{\text {Des }}^{\prime}(i, j)+(L+1) & P^{\prime}=-L, w(n)=1 \\ I_{\text {Des }}^{\prime}(i, j)+L & P^{\prime}=-L, w(n)=0 \\ I_{\text {Des }}^{\prime}(i, j)-L & P^{\prime}=L, w(n)=0,\end{cases}
$$




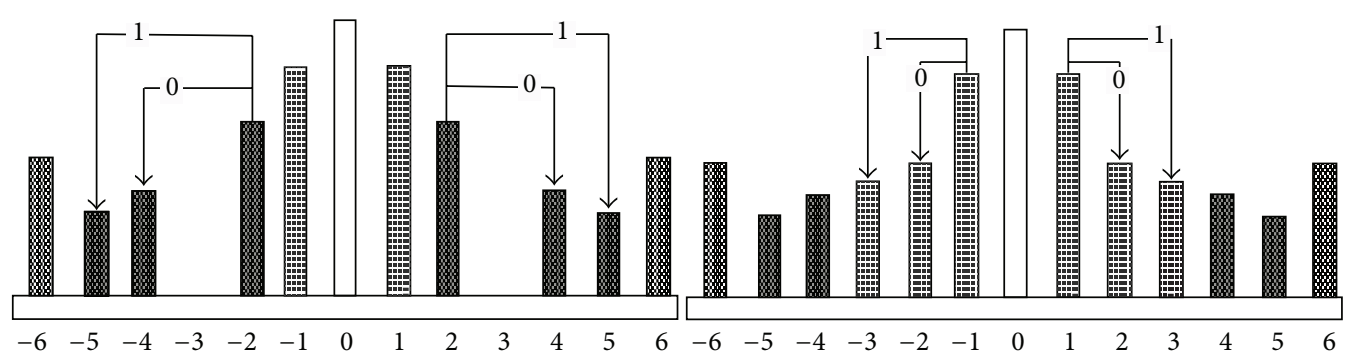

FIGURE 7: The moving process of histogram.
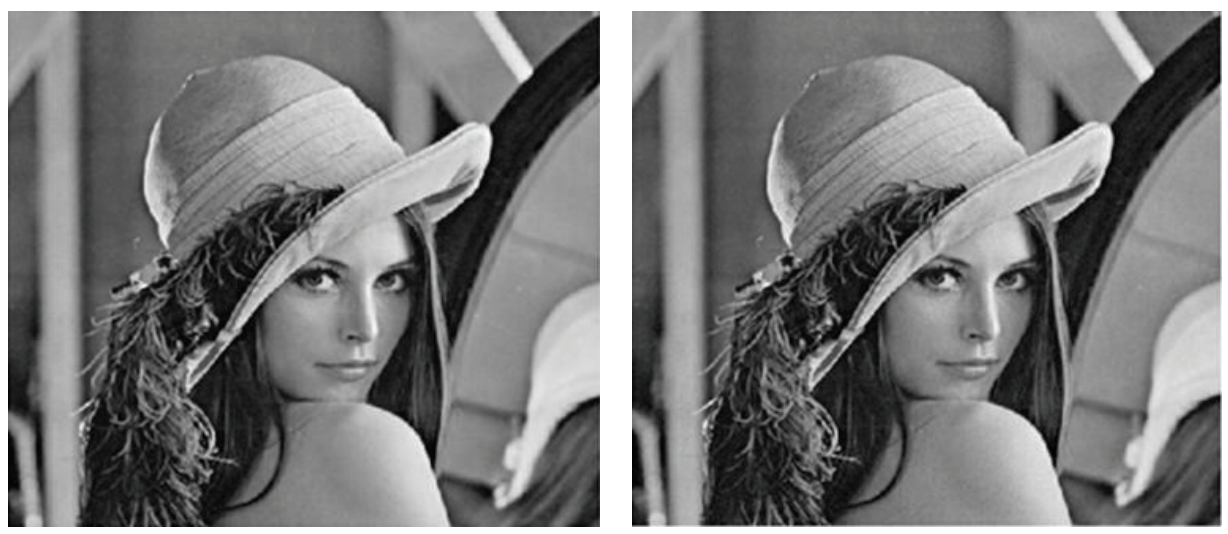

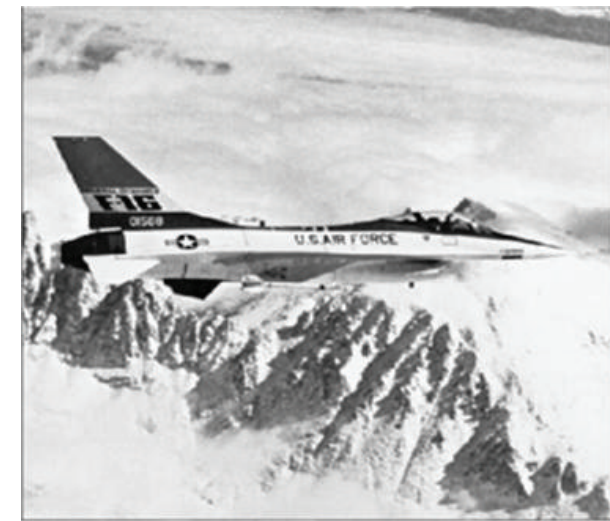

Cover image

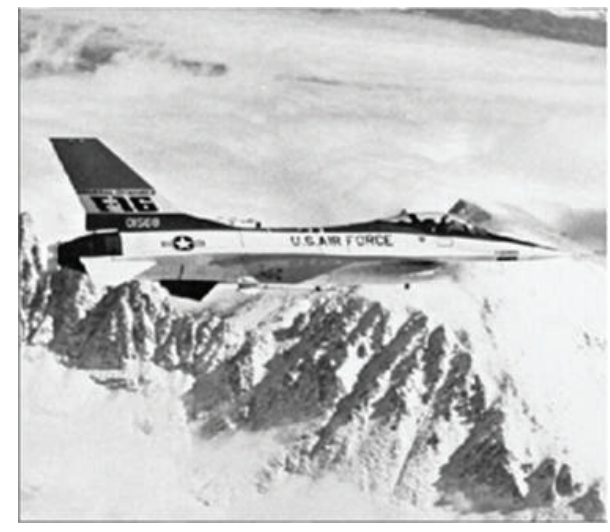

Stego-image

FIgURE 8: The cover images and the stego-images contain iris information.

$$
\begin{aligned}
& L=0, \\
& I_{\text {Des }}^{\prime \prime}(i, j)= \begin{cases}I_{\text {Des }}^{\prime}(i, j) & P^{\prime}=0, w(n)=1 \\
I_{\text {Des }}^{\prime}(i, j)-1 & P^{\prime}=0, w(n)=0 .\end{cases}
\end{aligned}
$$

The original image and the stego-image are shown in Figures 8 and 9. The extraction of secret information is an inverse process of the embedding process. First, the reference subsampled image is extracted from stego-image for computing the saliency image. Then, the stego-image is divided into blocks. The image blocks are sorted by the significance of image block. The reference subsampled image of image block is extracted by (3) and (4). The secret information is extracted by the secret key and the reference subsampled image. Finally, the biometric authentication image is reconstructed by residual image, dictionary, and sparse representation coefficients.

\section{Experiment and Analysis}

The proposed method is verified by the biometric authentication data in this paper, and the performance of the method is tested from security, invisibility, and embedding capacity.

3.1. Experimental Data. The PolyU multispectral palmprint database and the CASIA iris database of Chinese Academy of Sciences are used as biometric information $[24,25]$. We select 100 images from each database. The size of palmprint image 

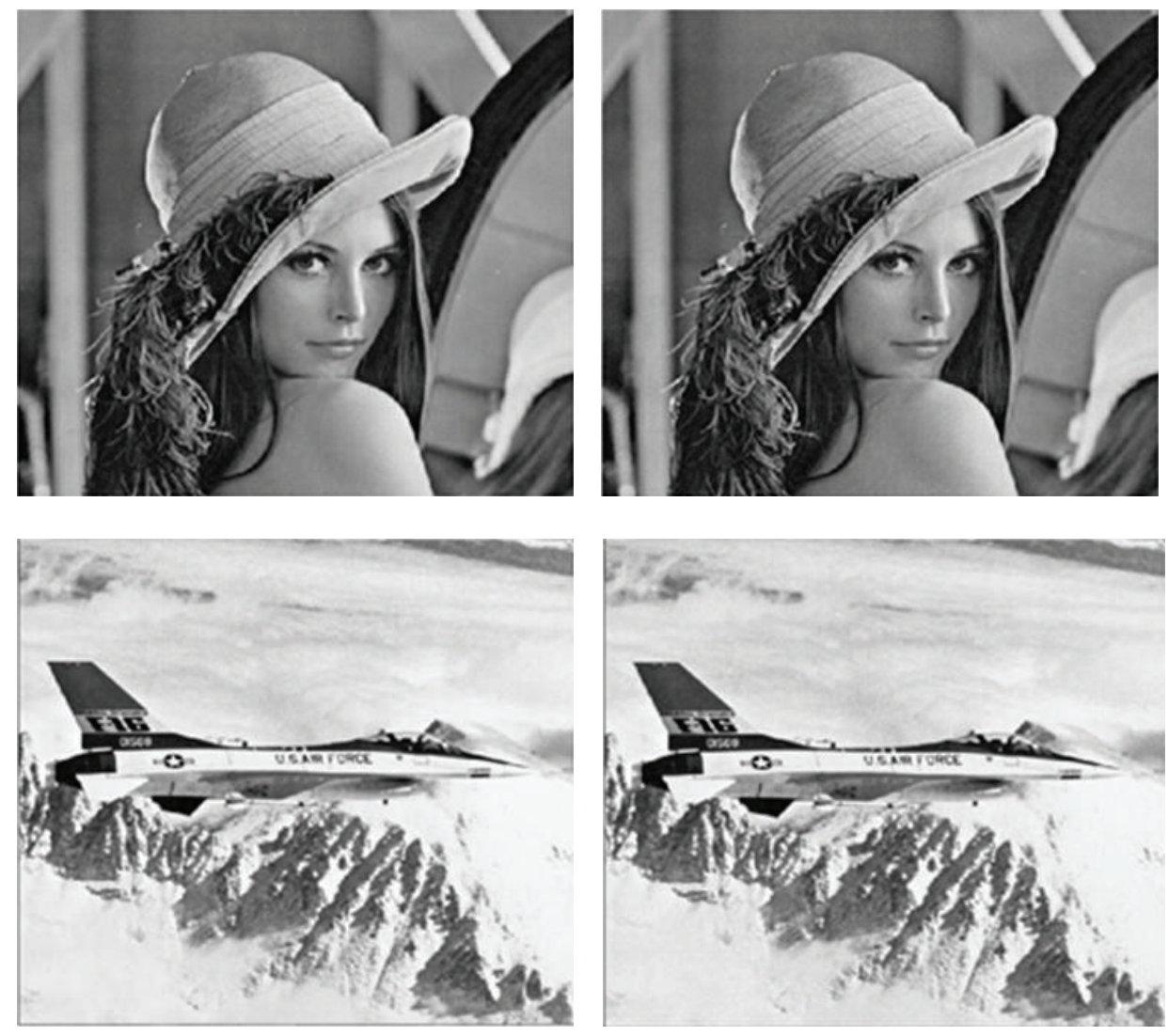

Cover image

Stego-image

FIgURE 9: The cover images and the stego-images contain palmprint information.
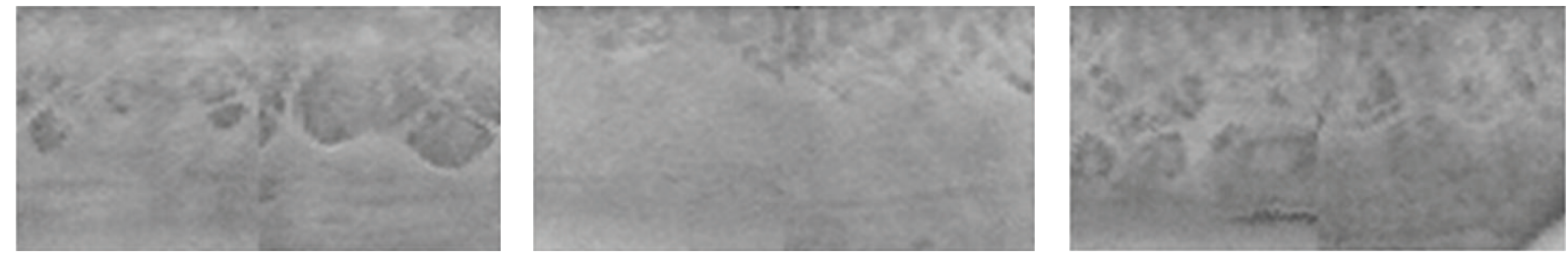

Figure 10: The iris images.

is $128 \times 128$; the size of iris image is $128 \times 64$. Some images in the database are shown in Figures 10 and 11. The cover images contain rich texture information and are unrelated with the biometric image. The cover images are shown in Figure 12.

3.2. Performance Analysis. Firstly, from the security point of view, the biometric information is hidden into the unnoticed cover image, which reduces the attacker's attention. Secondly, the main part of the biometric image is reconstructed by the sparse representation method, and the other part is embedded into the cover image. Even if the embedded information is intercepted, the attacker cannot restore the entire biometric image. Finally, the visual attention mechanism is used to select embedding location and embedding sequence of secret information. The visual attention mechanism increases the confidentiality of embedded information.
Peak Signal to Noise Ratio (PSNR) is an effective way to evaluate invisibility of information hiding. The PSNR of an image is calculated by the following:

$$
\operatorname{PSNR}=10 \times \log _{10}\left(\frac{255 \times 255 \times M \times N}{\sum_{i=1}^{M} \sum_{j=1}^{N}[C(i, j)-S(i, j)]^{2}}\right),
$$

where $C$ represents the cover image and $S$ represents the stego-image. When PSNR is higher than $30 \mathrm{~dB}$, we believe that the stego-image holds good invisibility. The proposed method and the method of literature [26] are compared and the comparison results are shown in Table 1.

From Table 1 we can see that the invisibility of our method is better than the other. Because the correlation analysis method based on sparse representation is used in the information hiding, the PSNR value of our method is higher 
TABLE 1: PSNR comparison results.

\begin{tabular}{lcccc}
\hline Cover image & Cover image (pixel) & Database & \multicolumn{2}{c}{ PSNR (dB) } \\
The proposed method
\end{tabular}
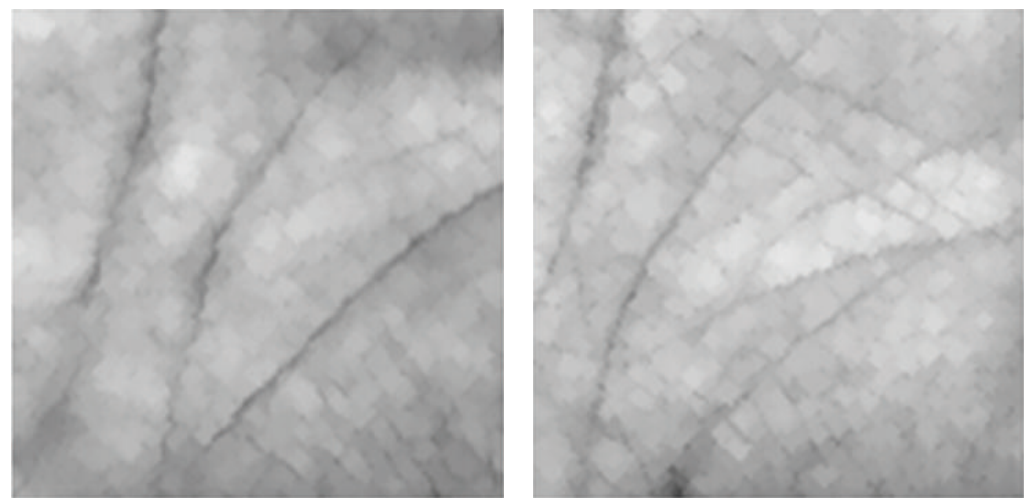

FIGURE 11: The palmprint images.
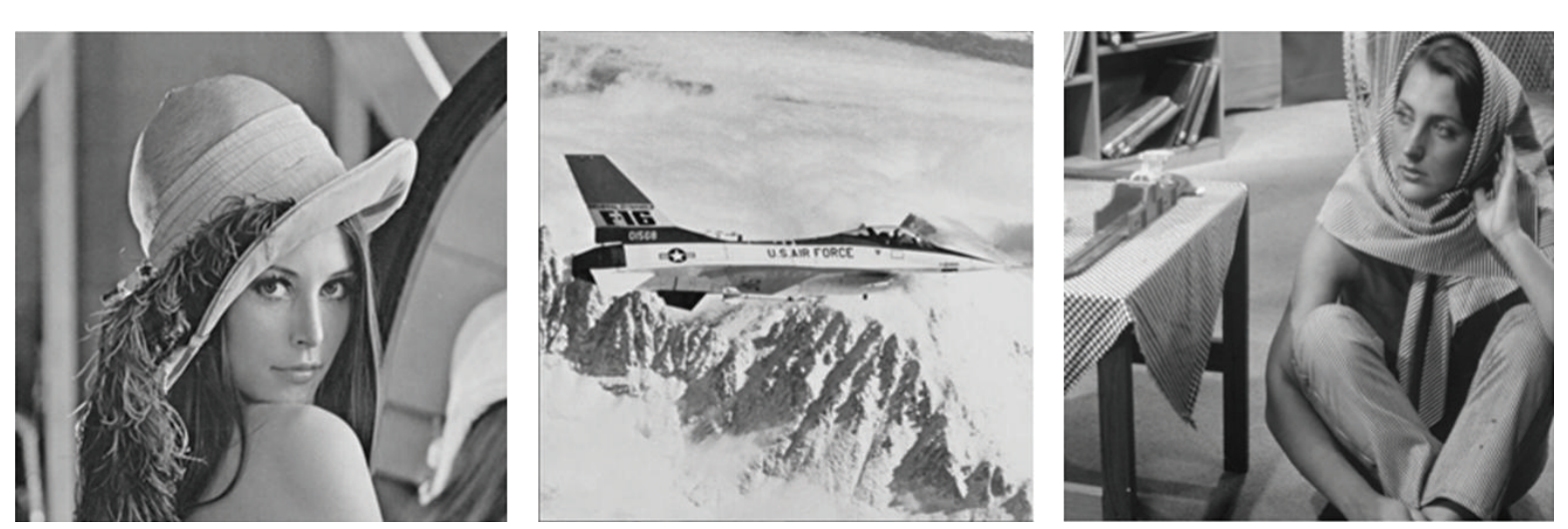

FIgURE 12: The cover images.
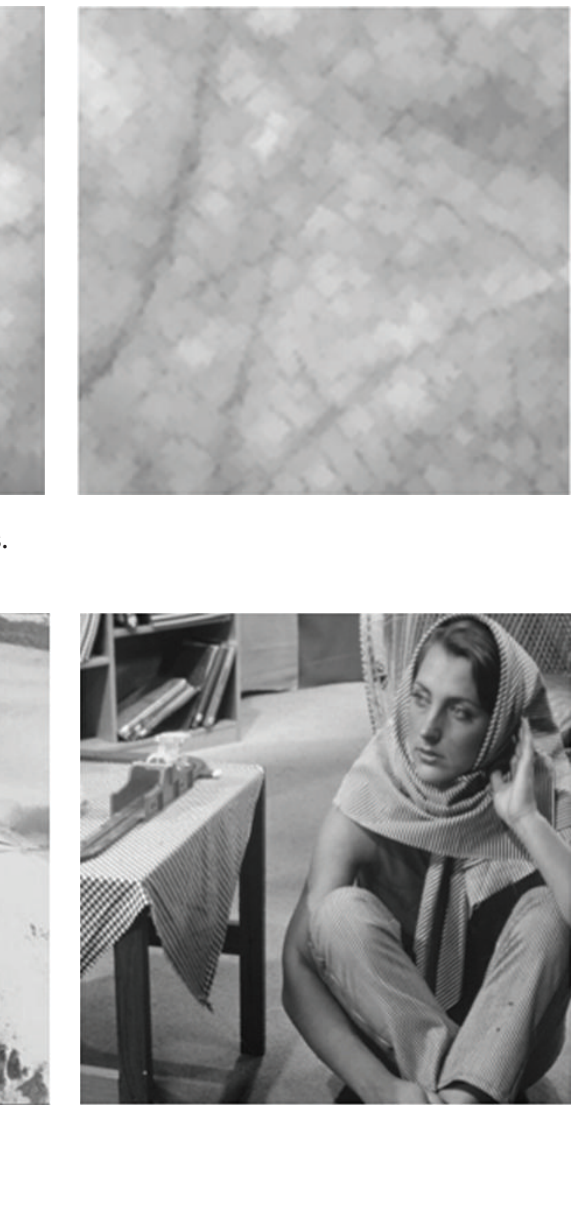

. 
TABLE 2: Embedding capacity comparison results.

\begin{tabular}{lccr}
\hline Algorithm & Cover image (pixel) & Secret information (bit) & bpp \\
\hline Vatsa et al. [27] & $1024 \times 768$ & 1000 & 0.0013 \\
Vatsa et al. [28] & $512 \times 512$ & 16384 & 0.0625 \\
Shih and Wu [29] & $512 \times 512 \times 3$ & 86016 & 0.1094 \\
Our method & $512 \times 512$ & 142084 & 0.5420 \\
\hline
\end{tabular}

Residual image is embedded into the cover image. In order to reduce the attacker's attention to stego-image, visual attention mechanism is used in the secret information embedding process. At the same time, the hiding strategy is modified in the information hiding algorithm, where the secret information embedding process is guided by the saliency image. Since the reconstruction method has a certain complexity, the secret key has high secrecy and is difficult to decipher. Because the biometric image is divided into two parts, the attacker cannot restore biometric authentication images with any part. A large number of experimental results show that the proposed method can protect the biometric authentication information effectively. Currently, our approach can only process the grayscale image. How to hide biometric information into color image is an important problem in future research.

\section{Conflict of Interests}

The authors declare that there is no conflict of interests regarding the publication of this paper.

\section{Acknowledgments}

This work is supported by the Science and Technology Research Project of Liaoning Province Education Department (no. L2014450), Social Science Planning Foundation Project of Liaoning Province (no. L13BXW006), Fund of Jilin Provincial Science \& Technology Department (nos. 20130206042GX and 20130522115JH), and National Natural Science Foundation of China (no. 61403078).

\section{References}

[1] P. Bedi, R. Bansal, and P. Sehgal, "Mulitimodal biometric authentication using PSO based watermarking," Procedia Technology, vol. 4, pp. 612-618, 2012.

[2] M. Vatsa, R. Singh, and A. Noore, "Feature based RDWT watermarking for multimodal biometric system," Image and Vision Computing, vol. 27, no. 3, pp. 293-304, 2009.

[3] A. K. Shaw, S. Majumder, S. Sarkar, and S. K. Sarkar, "A novel EMD based watermarking of fingerprint biometric using GEP," Procedia Technology, vol. 10, pp. 172-183, 2013.

[4] C. Li, Y. Wang, B. Ma, and Z. Zhang, “Tamper detection and self-recovery of biometric images using salient region-based authentication watermarking scheme," Computer Standards and Interfaces, vol. 34, no. 4, pp. 367-379, 2012.

[5] X. Che, J. Kong, J. Dai, Z. Gao, and M. Qi, "Content-based image hiding method for secure network biometric verification," International Journal of Computational Intelligence Systems, vol. 4, no. 4, pp. 596-605, 2011.
[6] M. Qi, Y. Lu, N. Du, Y. Zhang, C. Wang, and J. Kong, "A novel image hiding approach based on correlation analysis for secure multimodal biometrics," Journal of Network and Computer Applications, vol. 33, no. 3, pp. 247-257, 2010.

[7] Y. Shi, M. Qi, Y. Yi, M. Zhang, and J. Kong, "Object based dual watermarking for video authentication," Optik, vol. 124, no. 19, pp. 3827-3834, 2013.

[8] P. Jost, P. Vandergheynst, and P. Frossard, "Redundant image representations in security applications," in Proceedings of the International Conference on Image Processing (ICIP '04), pp. 2151-2154, October 2004.

[9] G. Cancelli, M. Barni, and G. Menegaz, "MPSteg: hiding a message in the matching pursuit domain," in Security, Steganography, and Watermarking of Multimedia Contents VIII, vol. 6072 of Proceedings of SPIE, pp. 1-9, 2006.

[10] G. Cancelli and M. Barni, "MPSteg-color: a new steganographic technique for color images," in Information Hiding: 9th International Workshop (IH '07), Saint Malo, France, June 11-13, 2007, vol. 4567 of Lecture Notes in Computer Science, pp. 1-15, Springer, Berlin, Germany, 2007.

[11] G. Cancelli and M. Barni, "MPSteg-color: data hiding through redundant basis decomposition," IEEE Transactions on Information Forensics and Security, vol. 4, no. 3, pp. 346-358, 2009.

[12] Y. C. Pati, R. Rezaiifar, and P. S. Krishnaprasad, "Orthogonal matching pursuit: recursive function approximation with applications to wavelet decomposition," in Proceedings of the 27th Annul Asilomar Conference on Signals, Systems and Computers, pp. 40-44, Pacific Grove, Calif, USA, 1993.

[13] S. G. Mallat and Z. Zhang, "Matching pursuits with timefrequency dictionaries," IEEE Transactions on Signal Processing, vol. 41, no. 12, pp. 3397-3415, 1993.

[14] M. W. Guo, Y. Z. Zhao, C. B. Zhang, and Z. H. Chen, "Fast object detection based on selective visual attention," Neurocomputing, vol. 144, no. 20, pp. 184-197, 2014.

[15] H. P. Yu, Y. X. Chang, P. Lu, Z. Y. Xu, C. Y. Fu, and Y. F. Wang, "Efficient object detection based on selective attention," Computers and Electrical Engineering, vol. 40, no. 3, pp. 907-919, 2014.

[16] Q. Liu, Q. Z. Zhang, W. B. Chen, and Z. C. Huang, "Pedestrian detection based on modeling computation of visual attention," Journal of Beijing Information Science \& Technology University, vol. 29, no. 2, pp. 59-65, 2014.

[17] C. Guo and L. Zhang, "A novel multiresolution spatiotemporal saliency detection model and its applications in image and video compression," IEEE Transactions on Image Processing, vol. 19, no. 1, pp. 185-198, 2010.

[18] C. Yang, L. H. Zhang, H. C. Lu, X. Ruan, and M.-H. Yang, "Saliency detection via graph-based manifold ranking," in Proceedings of the 26th IEEE Conference on Computer Vision and Pattern Recognition (CVPR '13), pp. 3166-3173, Portland, Ore, USA, June 2013. 
[19] B. Yang and S. T. Li, "Visual attention guided image fusion with sparse representation,” Optik, vol. 125 , no. 17 , pp. 4881-4888, 2014.

[20] H.-Y. Yang, Y.-W. Li, W.-Y. Li, X.-Y. Wang, and F.-Y. Yang, "Content-based image retrieval using local visual attention feature," Journal of Visual Communication and Image Representation, vol. 25, no. 6, pp. 1308-1323, 2014.

[21] X. H. Shen, Y. Wu, and Evanston, "A unified approach to salient object detection via low rank matrix recovery," in Proceedings of the IEEE Conference on Computer Vision and Pattern Recognition (CVPR '12), pp. 853-860, Providence, RI, USA, June 2012.

[22] K.-S. Kim, M.-J. Lee, H.-Y. Lee, and H.-K. Lee, "Reversible data hiding exploiting spatial correlation between sub-sampled images," Pattern Recognition, vol. 42, no. 11, pp. 3083-3096, 2009.

[23] J. H. Hwang, J. W. Kim, and J. U. Choi, "A reversible watermarking based on histogram shifting," in Digital Watermarking: 5th International Workshop (IWDW '06), Jeju Island, Republic of Korea, November 8-10, 2006, vol. 4283 of Lecture Notes in Computer Science, pp. 348-361, Springer, Berlin, Germany, 2006.

[24] PolyU multispectral palmprint Database, http://www.comp.polyu.edu.hk/ biometrics/MultispectralPalmprint/MSP.htm.

[25] CASIA-IrisV1[DB/OL], 2004, http://www.cbsr.ia.ac.cn/IrisDatabase.htm.

[26] M. Qi, J. Y. Dai, J. Z. Wang, X. X. Yu, and M. Zhang, "Contentbased reversible steganographic method for multimodal biometrics," Computer Science, vol. 39, no. 11, pp. 70-74, 2012.

[27] M. Vatsa, R. Singh, P. Mitra et al., "Comparing robustness of watermarking algorithms on biometrics data," in Proceedings of the Workshop on Biometric Challenges from Theory to Practice (ICPR Workshop '04), pp. 5-8, August 2004.

[28] M. Vatsa, R. Singh, A. Noore, M. M. Houck, and K. Morris, "Robust biometric image watermarking for fingerprint and face template protection," IEICE Electronics Express, vol. 3, no. 2, pp. 23-28, 2006.

[29] F. Y. Shih and S. Y. T. Wu, "Combinational image watermaking in the spatial and frequency domains," Pattern Recognition, vol. 36, no. 4, pp. 969-975, 2002. 


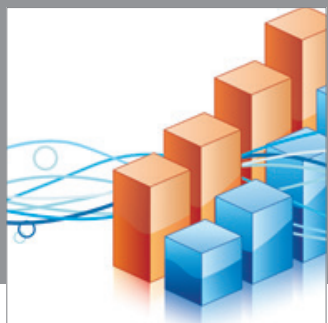

Advances in

Operations Research

mansans

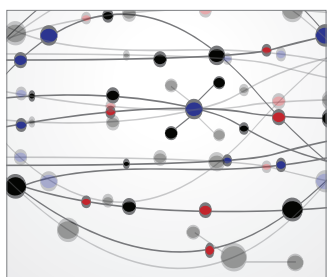

The Scientific World Journal
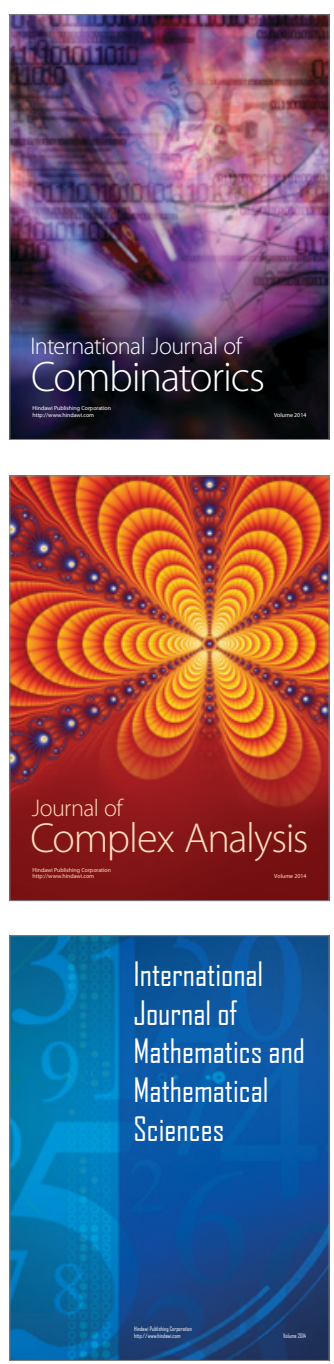
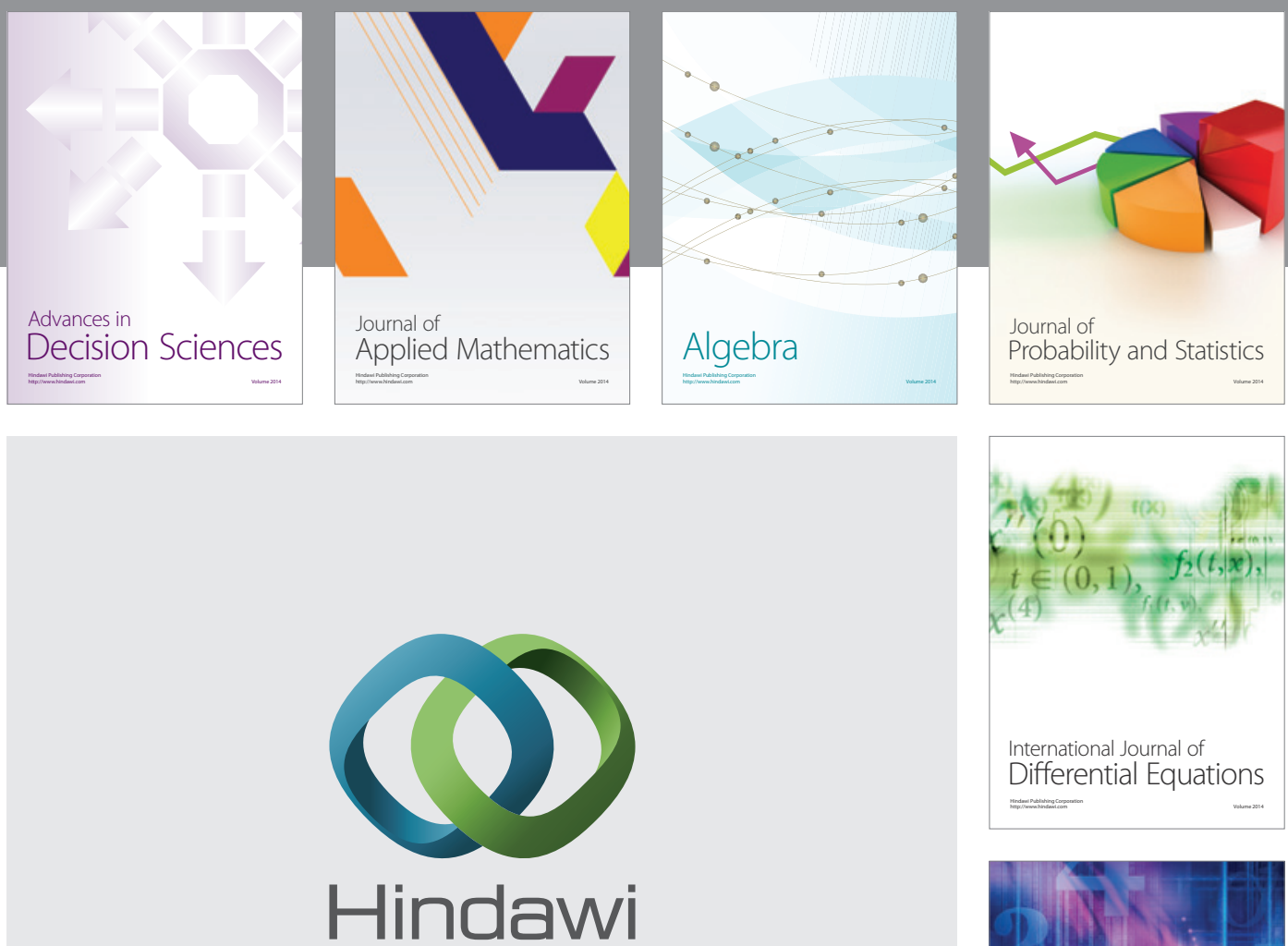

Submit your manuscripts at http://www.hindawi.com
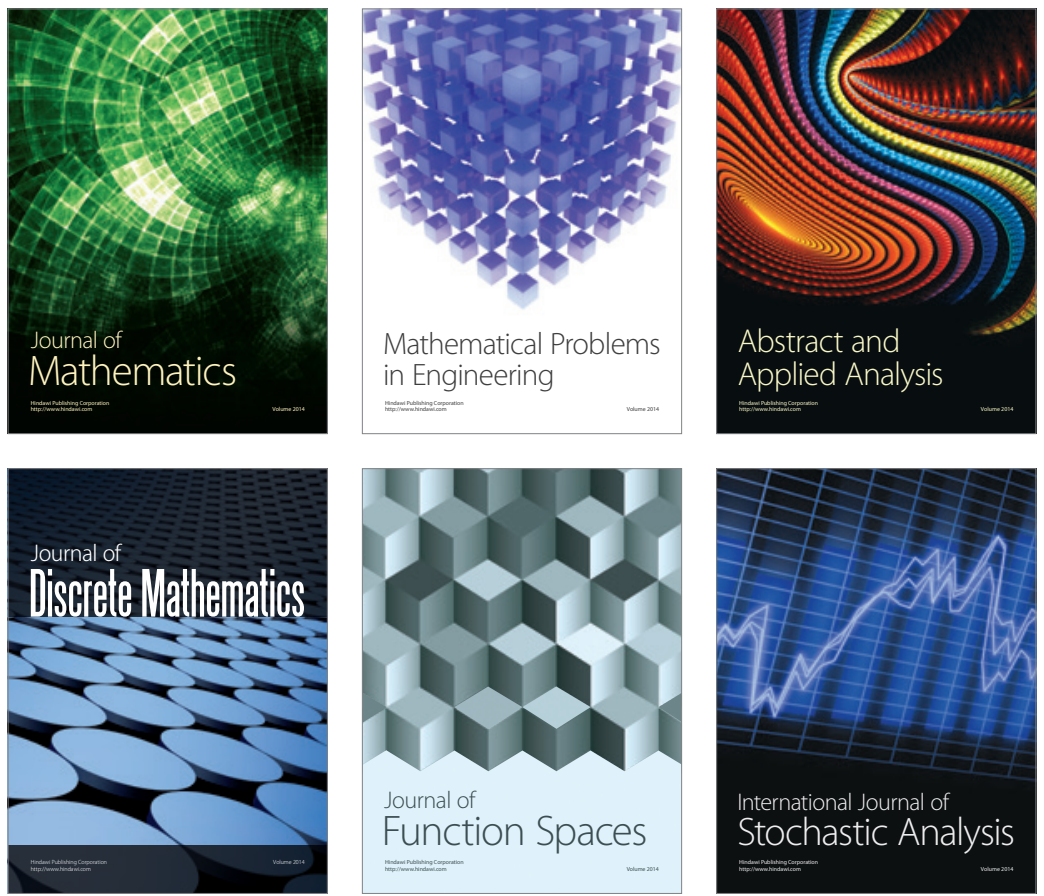

Journal of

Function Spaces

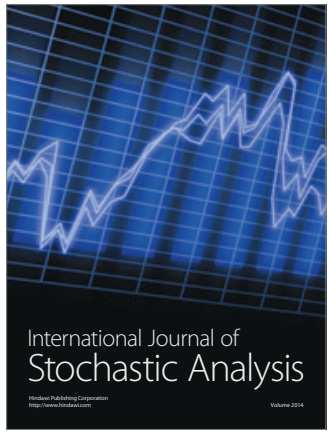

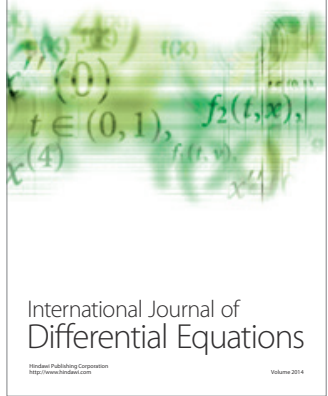
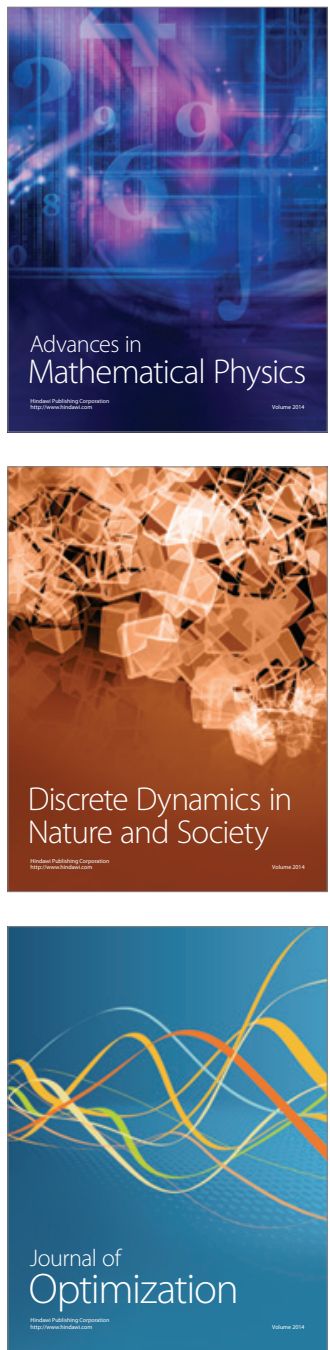